12

\title{
Влияние временной модуляции тока на величину трекинг-силы, действующей на релятивистский электронный пучок в омическом плазменном канале
}

\author{
(C) Е.К. Колесников, А.С. Мануйлов, В.С. Петров
}

Санкт-Петербургский государственный университет, 199034 Санкт-Петербург, Россия

ฯe-mail: a.manuylov@spbu.ru, man06@mail.ru

(Поступило в Редакцию 29 ноября 2017 г.)

В рамках модели „жесткого“ пучка исследовано влияние времени нарастания тока в импульсе релятивистского электронного пучка на трекинг-силу, действующую на пучок со стороны омического плазменного канала низкой проводимости. С помощью численного анализа показано, что увеличение времени нарастания тока пучка существенно уменьшает величину рассматриваемой трекинг-силы.

DOI: 10.21883/JTF.2018.07.46186.2576

\section{Введение}

Известно, что при распространении релятивистских электронных пучков (РЭП) в плотных газоплазменных средах вдоль омических плазменных каналов возникает проблема пучково-канального взаимодействия [1-9]. В работе [6] была сформулирована методика расчета трекинг-силы, притягивающей РЭП к предварительно созданному однородному по радиальному профилю омическому плазменному каналу достаточно низкой проводимости. Можно отметить, что в этой работе не был проведен численный анализ зависимости пучково-канальной трекинг-силы от скорости нарастания полного тока в импульсе пучка и временной зависимости трекинг-силы от амплитуды отклонения пучка от оси симметрии канала. Данным вопросам и посвящена настоящая работа.

\section{Постановка и решение задачи}

Рассмотрим параксиальный моноскоростной в продольном направлении азимутально-симметричный РЭП, распространяющийся вдоль оси $z$ цилиндрической системы координат $(r, \theta, z)$ вдоль предварительно созданного омического плазменного канала низкой скалярной проводимости $\sigma_{c h}$, когда выполнено условие $4 \pi \sigma_{c h} R_{b 0} / c \leq 1$, где $R_{b 0}-$ характерный радиус пучка, $c$ - скорость света. Будем предполагать, что проводимость $\sigma$ постоянна до некоторого радиуса $R_{c h}$ и равна $\sigma_{c h}$, а при $r>R_{c h}$ равна нулю. Предполагается, что при шланговых поперечных отклонениях пучок не выходит за пределы плазменного канала. При этом полагается, что динамика резистивной шланговой неустойчивости (РШН) ограничена только линейным режимом, когда выполнено соотношение $Y / R_{b 0} \ll 1$, здесь $Y$ - амплитуда поперечных отклонений оси симметрии пучка при развитиии указанной неустойчивости.
Используя методику, предложенную в работе [6], можно получить уравнение для определения временной зависимости дипольного электрического заряда, возникающего на границе однородного омического плазменного канала при поперечных отклонениях РЭП от оси канала в следующем виде:

$$
\frac{\partial \Re}{\partial \xi}+\frac{\Re}{2 \xi_{c}}=\frac{1}{2 \pi R_{c h}^{2} \xi_{c}} \int_{-\infty}^{\xi} d t \exp \left(\int_{\xi}^{t} \frac{d t_{1}}{\xi_{c}\left(t_{1}\right)}\right) \frac{\partial}{\partial t}\left(\frac{Y I_{b}}{c}\right)
$$

где $\Re$ - плотность дипольного поверхностного заряда на границе канала, $\xi=v_{z} \tau, \tau=t-z / \nu_{z}-$ сдвинутое время, $t-$ время, $z-$ дистанция, пройденная пучком, $v_{z}$ - продольная компонента скорости электронов пучка, $\xi_{c}=c /\left(4 \pi \sigma_{c h}\right)-$ характерная длина зарядовой нейтрализации, $I_{b}(\xi)=I_{b 0} k(\xi)$ - полный ток пучка, $I_{b 0}$ - характерный ток РЭП, $k(\xi)$ - заданная функция модуляции тока в импульсе пучка, которую выберем в виде

$$
k(\xi)=\operatorname{th}\left(\xi / \xi_{r}\right)
$$

где $\xi_{r}$ - характерная длина модуляции тока.

В результате решения уравнения (1) и подстановки в уравнение поперечной динамики РЭП в пределах плазменного канала, можно определить трекинг силу в виде

$$
F_{t r}=-F_{0} G(\xi, z),
$$

где $F_{0}=2 I_{b 0} R_{b 0} /\left(I_{A} R_{c h}^{2}\right)-$ характерное значение трекинг-силы, $I_{A}$ - предельный ток Альфвена,

$$
\begin{aligned}
G\left(\xi_{1}, z_{1}\right)= & -\int_{-\infty}^{\xi_{1}} d \xi_{1}^{*}\left[0.5 \exp \left(\frac{\xi_{1}^{*}-\xi_{1}}{2}\right)\right. \\
& \left.-\exp \left(\xi_{1}^{*}-\xi_{1}\right)\right] k\left(\xi_{1}^{*}\right) Y_{1}\left(z_{1}, \xi_{1}^{*}\right),
\end{aligned}
$$


$\xi_{1}=\xi / \xi_{c}, Y_{1}\left(z_{1}, \xi_{1}\right)=Y\left(z_{1}, \xi_{1}\right) / R_{b 0}$, где $Y\left(z_{1}, \xi_{1}\right)-$ амплитуда поперечного смещения оси симметрии тонкого поперечного сегмента пучка, расположенного на безразмерном расстоянии $\xi_{1}$ от фронта РЭП и прошедшего дистанцию $z\left(z_{1}=z k_{S}\right.$, где $k_{S}=\left(I_{b} / I_{A}\right)^{1 / 2} / R_{b 0}-$ бетатронное волновое число).

Предположим, что РШН пучка развивается вдоль импульса по закону

$$
Y_{1}\left(z_{1}^{*}, \xi_{1}\right)=Y_{0} \exp \left(a \xi_{1}\right) \cos \left(\omega \xi_{1}\right),
$$

где $Y_{0}-$ начальная амплитуда неустойчивости, $a-$ инкремент нарастания РШН вдоль импульса пучка, $\omega-$ временная частота колебаний при развитии неустойчивости, $z_{1}^{*}-$ некоторая фиксированная дистанция от ускорителя.

На рис. 1 представлена численная зависимость безразмерной силы $E_{t r} / F_{0}$ от координаты $\xi_{1}$ при значении временной частоты $\omega=0.5$ в случае, когда $a=0.2$, $Y_{0}=0.02$ и $k(\xi)=\operatorname{tr}\left(\xi / \xi_{r}\right)$. Кривая 1 соответствует характерному времени модуляции тока $\xi_{r}=0.2$, кривая $2-\xi_{r}=2,3-\xi_{r}=4$. Из рисунка видно, что в данной ситуации при $\xi_{1} \leq 4.5$ сила является отрицательной, т. е. возвращаюшей пучок обратно к оси симметрии неподвижного омического канала (является трекинг-силой). При больших $\xi_{1}$ сила меняет знак и становится отталкивающей пучок от канала (т.е. детрекинг-силой). Кроме того, из рисунка следует, что трекинг-сила убывает по величине с ростом характерного времени модуляции тока $\xi_{r}$.

На рис. 2 представлена рассчитанная зависимость безразмерной силы $F_{t r} / F_{0}=-G$ от величины $a-$ инкремента нарастания РШН вдоль импульса пучка при разных значениях характерного времени модуляции тока $\xi_{r}$. При этом выбраны следующие значения

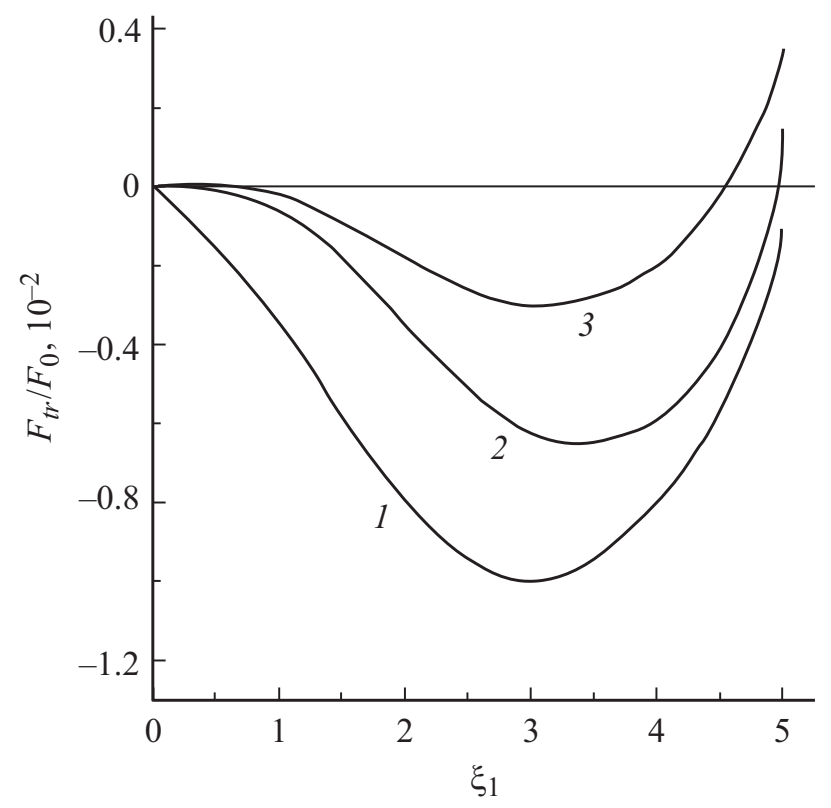

Рис. 1. Пояснение в тексте.

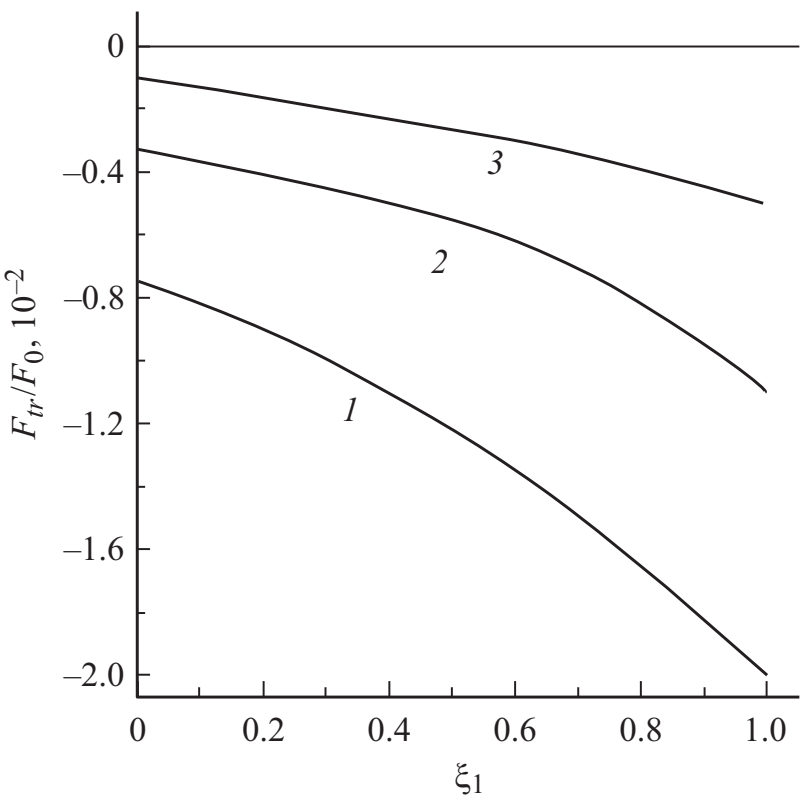

Рис. 2. Пояснение в тексте.

временной частоты $\omega$ и координаты $\xi_{1}: \omega=0, \xi_{1}=2$. Кривая 1 соответствует характерному времени модуляции тока $\xi_{r}=0.2$, кривая $2-\xi_{r}=2,3-\xi_{r}=4$. Из рис. 2 видно, что в данной ситуации сила является отрицательной (трекинг-силой). Кроме того, из рис. 2 следует, что трекинг-сила убывает по величине с ростом характерного времени модуляции тока $\xi_{r}$. Также можно отметить, что рассматриваемая сила увеличивается по модулю с ростом параметра $a-$ временного инкремента нарастания резистивной шланговой неустойчивости пучка.

\section{Заключение}

В настоящей работе рассмотрена задача о влиянии временной модуляции тока в импульсе релятивистского электронного пучка (РЭП) на трекинг-силу (возвращающую силу), действующую между пучком и омическим плазменным каналом при поперечном смещении пучка относительно оси симметрии канала, что имеет место при развитии резистивной шланговой неустойчивости РЭП. С помощью численного анализа при заданных законах изменения шланговой амплитуды и тока пучка вдоль его импульса показано, что увеличение характерного времени нарастания тока в импульсе РЭП существенно уменьшает величину силы пучково-канального взаимодействия. Кроме того, показано, что увеличение временного инкремента нарастания шланговой неустойчивости приводит к усилению изучаемой силы в несколько раз. 


\section{Список литературы}

[1] Рухадзе А.А., Богданкевич Л.С., Росинский С.Е., Рухлин В.Г. Физика сильноточных релятивистских электронных пучков. М.: Атомиздат, 1980. 167 с.

[2] Диденко А.Н., Григорьев В.П., Усов Ю.П. Мощные электронные пучки и их применение. М.: Атомиздат, 1977. $277 \mathrm{c}$.

[3] Миллер Р. Введение в физику сильноточных пучков заряженных частиц. М.: Мир, 1984. 432 с.

[4] Колесников Е.К., Мануйлов А.С., Филиппов Б.В. Динамика пучков заряженных частиц в газоплазменных средах. СПб.: Изд-во СПбГУ, 2002.98 с.

[5] Колесников Е.К., Мануйлов А.С., Зеленский А.Г. Динамика релятивистских электронных пучков в режиме ионной фокусировки. Воскресенск: Изд-во Позитив, 2013. 104 с.

[6] Lee E.P. // Livermore Lab. Rep. UCID-19675. 1983. 10 Janvary. $11 \mathrm{p}$.

[7] Hui B., Lampe M. // Proc. Fifth Intern. Conf. High Power Part. Beams. 1984. P. 374-377.

[8] Колесников Е.К., Мануйлов А.С. // ЖТФ. 1997. Т. 67. Вып. 6. C. 69-71.

[9] Мануйлов А.С. // ЖТФ. 2013. Т. 83. Вып. 10. С. 151-154. 